presence of a mobile stapes and absent or deficient incus. The KURZ Variac total prosthesis for use when the stapes is absent will be demontrated with the omega shoe connector. Malleus relocation combined with the Vincent ALTO prosthesis and the malleus replacement proshesis will also be shown. Data on results will be discussed with time for questions.

doi:10.1017/S0022215116002863

\section{Middle ear function in normal and pathological ears (K723)}

\section{ID: 723.1}

Middle Ear Function in Normal and Pathological Ears

Presenting Author: John Rosowski

John Rosowski

Massachusetts Eye and Ear Infirmary

Learning Objectives: - New measurements of TM motion tell us more about its function in normal and pathological ears. Besides being of use in diagnosing the presence of middle-ear effusion, WBI can aid in the diagnosis of ossicular and other conductive disorders. - Normal 'third-window's affect the response of the inner-ear to non-ossicularly conducted sound.

Introduction: In recent years multiple technical and research developments expanded our understanding of the workings of the normal and pathological ear: New measurements of normal and pathological tympanic-membrane (TM) function, new ways to asses ossicular disorders, and an improved understanding of the effect of cochlear 'third-windows'.

Methods: The clinic-based techniques of Wide-Band acoustic Immittance (WBI) and Laser-Doppler Vibrometry (LDV) are described along with experimental techniques: Digital Opto-Electronic Holography (DOEH), Optical Coherence Tomography (OCT), and inner-ear sound pressure measurements.

Results: WBI and scanning LDV and OCT shed new light on the function of the TM. DOEH, without scanning, reveals the temporal response of over 100,000 points on the TM surface, and demonstrates the complex modal response of the TM surface in response to frequencies as high as $20 \mathrm{kHz}$. The $\mathrm{TM}$ motions induced by sound of a few $\mathrm{kHz}$ and less assess the presence of multiple conductive disorders, including ossicular fixations or interruptions, as well as the presence of several cochlear conductive disorders. Recent clinical and basic research led to a re-evaluation of the 'two-window' model of the inner ear, which occurred in the presence of evidence for pathological 'third windows' that result from abnormalities in the bone around the innerear fluids.

Conclusions: Advances in measurement tools increased our understanding of the workings of the normal and pathological middle ear. (1) Spatially uniform sound-induced TM motions dominate the response to sound, and TM motions near the ossicular attachment contributes most to ossicular stimulation at high sound frequencies. (2) Multiple clinical techniques aid the pre-surgical diagnosis of ossicular and inner-ear conductive hearing disorders. (3) Normal inner-ear third windows explain multiple lines of evidence associated with non-ossicular stimulation of the inner ear.

doi:10.1017/S0022215116002875

\section{Balloon Tuboplasty (R731)}

\section{ID: 731.1}

\section{Site of Eustachian Tube Obstruction in} COM

\section{Presenting Author: Muaaz Tarabichi}

Muaaz Tarabichi, Muaaz Tarabichi

American Hospital Dubai

Learning Objectives: 1-Understand the existence of obstructive pathology in COM. 2-Learn how to evaluate for the site of obstruction. 3-Consider options for addressing obstruction within the proximal Eustachian tube.

Objective: To assess the patency of the proximal and distal segments of the Eustachian tube in patients undergoing surgery for chronic ear disease.

Study Design: Case study with control group.

Methods: All consecutive patients presenting for surgery for chronic ear disease in our practice over 14 months underwent preoperative Valsalva computed tomography (CT) and an attempt was made intraoperatively using angled rigid scopes to evaluate obstruction of the protympanic segment of the Eustachian tube. Endoscopic examination of the same segment in 19 cadaver ears served as a control group.

Results: Preoperative Valsalva CT showed patency of the distal one-third of the Eustachian tube in 51 of 53 ears. Intraoperative endoscopy allowed visualization of the protympanic opening of the Eustachian tube in 31/53 ears; $21 / 31$ ears showed obstruction of the protympanic opening of the Eustachian tube.

Conclusion: A clear obstruction was more likely to be present in the protympanic opening of the Eustachian tube in the patient population undergoing surgery for chronic ear disease than in the cadaver control group, and equally likely to be present in the distal cartilaginous tube in patients as in the control population.

doi:10.1017/S0022215116002887

\section{Balloon Tuboplasty (R731)}

\section{ID: 731.2}

Defining disease and outcome measures

Presenting Author: Mahmood Bhutta 